\title{
Influence of ultrasound-guided fine needle aspiration biopsy on cervical lymph node metastasis and prognosis of papillary thyroid microcarcinoma
}

\section{Ding Min}

Shanghai Jiao Tong University Medical School Affiliated Ruijin Hospital

\section{Zhifeng Zhao}

Shanghai Jiao Tong University School of Medicine Affiliated Ninth People's Hospital

\section{Dan Xu}

Shanghai Jiao Tong University Medical School Affiliated Ruijin Hospital

Tingyu Lin

Shanghai Jiao Tong University Medical School Affiliated Ruijin Hospital

\section{Dongjie Shen}

Ruijin Hospital Lu Wan Branch, Shanghai Jiaotong University School of Medicine

\section{Rongli Xie}

Ruijin Hospital/Lu Wan Branch, Shanghai Jiaotong University School of Medicine

Jian Fei ( $\nabla$ fj10777@rjh.com.cn)

Shanghai Jiao Tong University Medical School Affiliated Ruijin Hospital https://orcid.org/0000-00017159-8562

\section{Research Article}

Keywords: papillary thyroid carcinoma, microcarcinoma, fine-needle aspiration biopsy, cervical lymph node metastasis, prognostic factor

Posted Date: November 8th, 2021

DOI: https://doi.org/10.21203/rs.3.rs-1036349/v1

License: (c) (1) This work is licensed under a Creative Commons Attribution 4.0 International License. Read Full License 


\section{Abstract \\ Purpose}

To analyze the potential association between UGFNA and cervical lymph node metastasis (CLNM) of papillary thyroid microcarcinoma (PTMC) and whether it would influence the long-term prognosis of PTMC. Then we could give advice for the application of UGFNA for thyroid nodules less than $1 \mathrm{~cm}$.

\section{Methods}

A total of 367 adult patients with PTMC who underwent thyroidectomy in our hospital during January 2014 and December 2015 were enrolled in this retrospective cohort study. Univariate and multivariate analysis were conducted to explore the potential risk factors of CLNM. Propensity score (PS) matching was performed to select patients with homogeneous baseline characteristics. Then the rate of CLNM was compared in matched samples by chi-squared $(\mathrm{X} 2)$. Survival analysis was made by Kaplan-Meier method in PS-matching cohort.

\section{Results}

CLNM rate in PS-matching cohort showed no significant difference between UGFNA group and nonUGFNA group. (29.5\% vs $23.7 \%, P>0.05)$. Multivariate analysis showed that male sex $(O R=3.997,95 \% \mathrm{Cl}$ 2.148 7.439, $\mathrm{P}<0.01)$, larger tumor size $(\mathrm{OR}=2.319,95 \% \mathrm{Cl} 1.285 \sim 4.186, \mathrm{P}<0.01)$ were independent risk factors of CLNM. With a median follow-up of 63.8 months (range 3.1-84.9 months), the 5-year cumulative recurrence-free survival (RFS) rate was $99.3 \%$ in UGFNA group and $97.7 \%$ in non-UGFNA group. After PSmatching, Kaplan-Meier curves showed no significant difference from each other $(P>0.05)$.

\section{Conclusions}

UGFNA was a very safe diagnostic tool, which would not influence the CLNM rate and long-term prognosis of patients with PTMC. Regardless of the costs, we recommend routine UGFNA for suspicious thyroid nodules $\leqq 1 \mathrm{~cm}$.

\section{Introduction}

Over the last several decades, incidence of thyroid cancer (TC) has increased rapidly in both men and women globally[1]. Papillary thyroid carcinoma (PTC) was the most common and indolent type of TC, which accounted for $80 \%$ of the total TC cases[1-3]. Moreover, most of the newly diagnosed cancer type was papillary thyroid microcarcinoma (PTMC) whose size is $1 \mathrm{~cm}$ or less $[4,5]$ due to the increasing use of medical imaging such as ultrasounds and computed tomography scan[6, 7]. 
Early diagnosis is very important for PTC patients as its 10-year survival rate can be as high as $90 \%$ in early stage, while decreased significantly in later stage[3]. Ultrasound guided fine needle aspiration biopsy (UGFNA) is the most common and cost-effective diagnostic method for TC, which help doctors distinguish malignant nodules from benign ones before the surgery and aid in treatment decisionmaking[8, 9]. Some complications associated with UGFNA such as pain, bleeding, recurrent laryngeal nerve palsy, needle tract implantation (NTI) could also be observed in clinical practice, although the occurrence rate was low (10). Patients may still warry about UGFNA associated tumor dissemination. To our knowledge, the prognostic influence of UGFNA on patients with PTMC had not been studied systematacially before. In this study, we retrospectively analyzed the relationship between UGFNA and cervical lymph node metastasis (CLNM) and whether it would influence the long-term prognosis of patients with PTMC. Then we could give advice for the application of UGFNA for thyroid nodules less than $1 \mathrm{~cm}$.

\section{Materials And Methods}

\section{Patients}

UGFNA for thyroid nodules was gradually carried out in clinical practice as a new preoperative diagnostic tool in 2015 in China. Since then, more and more patients preferred to choose preoperative UGFNA to learn about the nature of thyroid nodules. So we selected patients who underwent thyroidectomy during January 2014 and December 2015 in our Hospital as objects of our study. The use of UGFNA was based on patients' willingness and evaluation of nodules by US. All patients were confirmed as PTMC by postoperative pathology. Finally, a total of 367 adult patients were enrolled in this retrospective cohort study. Patients were divided into two groups according to whether underwent preoperative UGFNA. There are 163 cases in UGFNA group and 204 cases in non-UGFNA group respectively.

\section{Data collection}

The electronic medical records and surgical pathology reports of each patient were reviewed to define the demographic characteristics and clinicopathological features, including multifocality, capsular invasion, Hashimoto's thyroiditis (HT), tumor size, CLNM, operation method and cervical lymph node dissection. The size of tumor was measured by postoperative pathology. As for multifocal tumor, only the largest diameter among all nodules was selected to be analyzed. Cervical lymph node dissection was stratified by whether performed central lymph node dissection. All operations were performed by the same surgery group of our hospital and pathological analysis was then taken by the department of pathology. The data of follow-up were collected by retrospective review of the patients' medical records and telephone interview. The last follow-up was on January 10, 2021. Recurrence was recorded when there were suspicious cervical lymph nodes or residual thyroid nodes which were further confirmed malignant by UGFNA or pathology of secondary surgery. Tumor recurrence was used as the end point of the analysis to assess the outcome. Recurrence-free survival (RFS) time was calculated in months from the date of first surgery to date of recurrence or last follow-up. 


\section{Statistical analysis}

Statistical analysis was performed by IBM SPSS Statistics Version.23. The data was expressed as median for continuous variables and percentages for categorical variables. Comparisons between two groups were performed by Mann-Whitney $U$ test for continuous variables and chi-squared $(\chi 2)$ for categorical variables. In addition, univariate logistic and multivariate logistic regression were performed to explore the relationship between the clinical pathological features and CLNM in patients who underwent cervical lymph node dissection. Propensity score (PS) of each patient was calculated by logistic regression models by entering the following covariables: age group, gender, operative method, lymph node dissection, capsular invasion, tumor size and multifocality. Then the PS-matching (1:1 nearest neighbor matching) was performed by using a caliper of 0.2 standard deviation to minimize the differences in covariates between two groups. The survival curves were plotted by Kaplan-Meier method. RFS rate was compared across groups by Log-rank test in PS-matching cohort. $P$ values less than 0.05 was considered to be statistically significant. ( $*$ : $P<0.05, * *$ : $P<0.01)$

\section{Results}

\section{Comparisons of baseline characteristics in PTMC patients between UGFNA group and non-UGFNA group before PS-matching}

A total of 367 patients were enrolled in this study. The median age of PTMC patients was 47 years with a range of 22-76 years. The median age in UGFNA group was significantly lower than that in non-UGFNA group ( $P=0.041$ ). After dividing patients into two groups by the age cutoff of 55 years, no significant difference in distribution of age was found between the two groups $(P=0.588)$. Among all patients, $71.9 \%$ of them were female, and no significant difference in proportion of female was found between the two categories (71.8\% vs72.1\%; $P=0.953)$. Also, clinicopathological characteristics of patients with PTMC including multifocality $(P=0.785)$, capsular invasion $(P=0.091)$, tumor size $(P=0.812)$ and $\mathrm{HT}(P=0.678)$ all showed no significant difference between the two categories. Operative method also had no significant difference in two groups $(P=0.168)$. Interestingly, the rate of cervical lymph node dissection in UGFNA group was significantly higher than that in non-UGFNA group (87.1\% vs 61.3\%; $P<0.01)$. (Table 1)

\section{UGFNA showed no significant influence on CLNM in PS-matching cohort}

To balance the heterogeneity of baseline characteristics between UGFNA group and non-UGFNA group, PS-matching (1:1 nearest neighbor matching) was taken with a caliper of 0.2 standard deviation. After PS-matching, there were 139 matched PTMC patients in each group. And all baseline characteristics, including cervical lymph node dissection, showed no significant difference between the two groups after PS-matching. (Table 2) In the PS-matching cohort, the rate of CLNM was 29.5\% in UGFNA group and $23.7 \%$ in non-UGFNA group, which showed no significant difference from each other. $(\mathrm{OR}=1.344,95 \% \mathrm{Cl}$ $0.788 \sim 2.293, P=0.278$ ) 
As the rate of cervical lymph node dissection had a significant difference between two groups. Only patients who underwent cervical lymph node dissection were selected to analyze the risk factors of CLNM in PTMC. In univariate logistic regression analysis, we found that CLNM in patients with PTMC were associated with male sex $(\mathrm{OR}=3.504,95 \% \mathrm{Cl} 1.984 \sim 6.188, P<0.01)$ and larger tumor size $(\mathrm{OR}=2.105$, $95 \% \mathrm{Cl} 1.229 \sim 3.605, P<0.01)$. Furthermore, after adjusting confound variables by performing multivariate logistic regression analysis, male sex $(\mathrm{OR}=3.997,95 \% \mathrm{Cl} 2.148 \sim 7.439, P<0.01)$, larger tumor size $(\mathrm{OR}=2.319,95 \% \mathrm{Cl} 1.285 \sim 4.186, P<0.01)$ were still independent risk factors of CLNM. UGFNA was not independent risk factors of UGFNA, which was consist with the above result in PS-matching cohort. (Table 3)

\section{Follow-up and prognosis}

The median follow-up time was 63.8 months (range $3.1-84.9$ months). $13.4 \%$ of the patients were lost to follow up at the beginning. Six patients, 2 patients in UGFNA group and 4 patients in non-UGFNA group, showed disease recurrence in the follow-up visit. The 5-year cumulative RFS rate was $99.3 \%$ in UGFNA group and $97.7 \%$ in non-UGFNA group. In PS-matching cohort, Kaplan-Meier curves showed no significant difference between the two groups ( $P=0.927)$ (Fig.1). Therefore, UGFNA would not influence patients' long-term prognosis.

\section{Discussion}

Although UGFNA is generally recognized as a safe diagnostic tool. In clinical practice, patients may still worry about the adverse effect of UGFNA especially UGFNA associated metastasis. The most commonly reported UGFNA associated metastasis was NTI in previous study. The cumulative incidence rate of NTI in TC were $0.15 \%$ and $0.37 \%$ at 5 and 10 years after FNA. [10] However, the impacts of UGFNA on CLNM and long-term prognosis of PTMC were still unknow. In our study, we demonstrate that UGFNA was not risk factor of CLNM nor tumor recurrence.

It is still controversial for the employment of UGFNA on thyroid nodules less than $1 \mathrm{~cm}$. The 2015 ATA Management Guidelines and the 2017 ACR Thyroid Imaging, Reporting and Data System (TI-RADS) both set size cutoff of UGFNA only at $\geqq 1 \mathrm{~cm}$ even for high suspicious nodules. UGFNA was not recommend for nodules less $1 \mathrm{~cm}$ unless there were ultrasonographic or clinical malignant signs such as extracapsular extension, indeterminate cervical lymph nodes, a history of neck radiation $[9,11,12]$. However, some PTMC also tend to be aggressive which may be misdiagnosed if they didn't receive preoperative UGFNA. In this study, we demonstrated the safety of UGFNA for PTMC, so we recommend preoperative UGFNA for suspicious thyroid nodules less than $1 \mathrm{~cm}$ when patients had the willingness even if they didn't meet the recommend criteria of guidelines.

Moreover, we found that male sex, larger tumor size were independent risk factors of lymph node metastasis as it has been reported before[13]. As the eighth AJCC/TNM cancer staging system raised the age cutoff of stage from 45 to 55 years, our study selected 55 years as age cut-off which was different from previous studies[14]. Younger age, capsular invasion and multifocality were neither the independent 
risk factors of CLNM, although the significance has been demonstrated on previous studies[13]. HT was also not related with CLNM in our study, researchers haven't reached an agreement on whether HT was a risk factor for CLNM[15].

There are still some limitations in our study. First, the enrolled members were not large enough with only 367 cases included. As patients with PTMC had a relatively low recurrence rate, more patients and longer follow-up visit were needed. Second, this was a single-center analysis, there might been some selection bias, multi-center study was needed to further confirm our results. Third, although the PS-matching method was performed to balance the heterogeneity of baseline characteristics, there were still some variables which were not included in this study. Therefore, their potential confounding effects on the results could not be adjusted unavoidably.

In conclusion, this study indicated that UGFNA won't influence the CLNM and long-term prognosis of patients with PTMC, which was considered as a relatively safe preoperative diagnosed tool. Regardless of the costs, we recommend routine UGFNA for suspicious thyroid nodules less than or equal to $1 \mathrm{~cm}$.

\section{Declarations}

\section{Data availability}

The data sets used and/or analyzed in the present study are available from the corresponding authors upon request.

\section{Compliance with Ethical Standards}

\section{Conflict of interest}

The authors have no relevant financial or non-financial interests to disclose.

\section{Ethical approval}

Approval was obtained from the ethics committee of Shanghai Jiao Tong University Medical School Affiliated Ruijin Hospital, Shanghai, China. The procedures used in this study adhere to the tenets of the Declaration of Helsinki.

\section{Inform consent}

Since this was a retrospective research, informed consent was waived by our institutional ethic committee.

\section{References}

1. C. La Vecchia, M. Malvezzi, C. Bosetti et al., Thyroid cancer mortality and incidence: a global overview. International journal of cancer. 136(9):2187-95. doi:10.1002/ijc.29251 
2. H. Lim, S.S. Devesa, J.A. Sosa, D. Check, C.M. Kitahara, Trends in Thyroid Cancer Incidence and Mortality in the United States, 1974-2013. Jama. 317(13):1338-1348. doi:10.1001/jama.2017.2719

3. J. Lu, S. Hu, P. Miccoli et al., Non-invasive diagnosis of papillary thyroid microcarcinoma: a NMRbased metabolomics approach. Oncotarget. 7(49):81768-81777. doi:10.18632/oncotarget.13178

4. C. Hedinger, E.D. Williams, L.H. Sobin, The WHO histological classification of thyroid tumors: a commentary on the second edition. Cancer. 63(5):908-11. doi:10.1002/10970142(19890301)63:5<908::aid-cncr2820630520>3.0.c0;2-i

5. L. Davies, H.G. Welch, Increasing incidence of thyroid cancer in the United States, 1973-2002. Jama. 295(18):2164-7. doi:10.1001/jama.295.18.2164

6. S.R. Baker, W.A. Bhatti, The thyroid cancer epidemic: is it the dark side of the CT revolution? European journal of radiology. 60(1):67-9. doi:10.1016/j.ejrad.2006.04.022

7. W.D. Kent, S.F. Hall, P.A. Isotalo, R.L. Houlden, R.L. George, P.A. Groome, Increased incidence of differentiated thyroid carcinoma and detection of subclinical disease. CMAJ: Canadian Medical Association journal = journal de l'Association medicale canadienne.177(11):1357-61. doi:10.1503/cmaj.061730

8. Y.E. Nikiforov, D.L. Steward, T.M. Robinson-Smith et al., Molecular testing for mutations in improving the fine-needle aspiration diagnosis of thyroid nodules. The Journal of clinical endocrinology and metabolism. 94(6):2092-8. doi:10.1210/jc.2009-0247

9. B.R. Haugen, E.K. Alexander, K.C. Bible et al., 2015 American Thyroid Association Management Guidelines for Adult Patients with Thyroid Nodules and Differentiated Thyroid Cancer: The American Thyroid Association Guidelines Task Force on Thyroid Nodules and Differentiated Thyroid Cancer. Thyroid: official journal of the American Thyroid Association. 26(1):1-133. doi:10.1089/thy. 2015.0020

10. T. Hayashi, M. Hirokawa, M. Higuchi, T. Kudo, Y. Ito, A. Miyauchi, Needle Tract Implantation Following Fine-Needle Aspiration of Thyroid Cancer. World journal of surgery.44(2):378-384. doi:10.1007/s00268-019-05161-1

11. J. Feldkamp, D. Führer, M. Luster, T.J. Musholt, C. Spitzweg, M. Schott, Fine Needle Aspiration in the Investigation of Thyroid Nodules. Deutsches Arzteblatt international.113(20):353-9. doi:10.3238/arztebl.2016.0353

12. F.N. Tessler, W.D. Middleton, E.G. Grant et al. ACR Thyroid Imaging, Reporting and Data System (TIRADS): White Paper of the ACR TI-RADS Committee. Journal of the American College of Radiology: JACR. 14(5):587-595. doi:10.1016/j.jacr.2017.01.046

13. L. Zhang, W.J. Wei, Q.H. Ji et al., Risk factors for neck nodal metastasis in papillary thyroid microcarcinoma: a study of 1066 patients. The Journal of clinical endocrinology and metabolism. 97(4):1250-7. doi:10.1210/jc.2011-1546

14. R.M. Tuttle, B. Haugen, N.D. Perrier, Updated American Joint Committee on Cancer/Tumor-NodeMetastasis Staging System for Differentiated and Anaplastic Thyroid Cancer (Eighth Edition): What 
Changed and Why? Thyroid: official journal of the American Thyroid Association. 27(6):751-756. doi:10.1089/thy.2017.0102

15. H.Y. Bircan, B. Koc, C. Akarsu et al., Is Hashimoto's thyroiditis a prognostic factor for thyroid papillary microcarcinoma? European review for medical and pharmacological sciences. 18(13):1910-5

\section{Tables}

Table 1 Comparison of clinicopathological characteristics in PTMC patients between UGFNA group and nonUGFNA group

\begin{tabular}{|c|c|c|c|c|c|c|}
\hline \multirow[t]{2}{*}{ Characteristics } & Total & \multicolumn{2}{|c|}{ UGFNA $^{\mathrm{a}}(+)$} & \multicolumn{2}{|c|}{ UGFNA (-) } & \multirow[t]{2}{*}{$P$ value } \\
\hline & $(\mathrm{N}=367)$ & $(\mathrm{N}=163)$ & $\mathrm{N}(\%)$ & $(\mathrm{N}=204)$ & $\mathrm{N}(\%)$ & \\
\hline Age (years) & & & & & & 0.588 \\
\hline$<55$ & $249(67.8)$ & $113(6$ & & 136( & & \\
\hline$\geqq 55$ & $118(32.2)$ & $50(3$ & & $68(3$ & & \\
\hline Female sex & 264(71.9) & $117(7$ & & 147( & & 0.953 \\
\hline Multifocality & $52(14.2)$ & $24(1$ & & $28(1$ & & 0.785 \\
\hline Capsular invasion & $19(5.2)$ & $12(7$ & & $7(3$ & & 0.091 \\
\hline Tumor size $(\mathrm{cm})$ & & & & & & 0.812 \\
\hline$\leqq 0.5$ & $243(66.2)$ & 109(6 & & 134( & & \\
\hline$>0.5$ & $124(33.8)$ & $54(3$ & & 700 & & \\
\hline $\mathrm{HT}^{\mathrm{b}}$ & $73(19.9)$ & $34(2$ & & $49(1$ & & 0.678 \\
\hline Operative method & & & & & & 0.168 \\
\hline $\mathrm{LT}^{\mathrm{c}}$ & $245(66.8)$ & $115(7$ & & 130( & & \\
\hline $\mathrm{TT}^{\mathrm{d}}$ & $122(33.2)$ & $48(2$ & & $74(3$ & & \\
\hline Cervical lymph node dissection & $267(72.8)$ & $142(8$ & & 125( & & $0.000 * *$ \\
\hline
\end{tabular}

a. UGFNA= Ultrasound-guided fine needle aspiration biopsy; b. HT= Hashimoto's thyroiditis; c. LT=Lobectomy; d.TT=Total thyroidectomy. **: $\mathrm{P}<0.01$

Table 2 Comparison of clinicopathological characteristics in PTMC patients between UGFNA group and nonUGFNA group after PS-matching 


\begin{tabular}{|c|c|c|c|c|c|}
\hline \multirow[t]{3}{*}{ Characteristics } & \multicolumn{2}{|l|}{ Total } & UGFNA $^{\mathrm{a}}(+)$ & UGFNA (-) & \multirow[t]{3}{*}{$P$ value } \\
\hline & $(\mathrm{N}=278)$ & $\mathrm{N}$ & $(\mathrm{N}=139)$ & $(\mathrm{N}=139)$ & \\
\hline & $(\%)$ & & $(\%)$ & $(\%)$ & \\
\hline Age (years) & & & & & \multirow[t]{2}{*}{0.250} \\
\hline$<55$ & $187(67.3)$ & & $89(64.0)$ & $98(70.5)$ & \\
\hline$\geqq 55$ & $91(32.7)$ & & $50(36.0)$ & $41(29.5)$ & \\
\hline Female sex & $195(70.1)$ & & $95(68.3)$ & $100(71.9)$ & 0.512 \\
\hline Multifocality & $46(16.5)$ & & $24(17.3)$ & $22(15.8)$ & 0.747 \\
\hline Capsular invasion & $16(5.8)$ & & $9(6.5)$ & $7(5.0)$ & 0.607 \\
\hline Tumor size $(\mathrm{cm})$ & & & & & 0.381 \\
\hline$\leqq 0.5$ & $179(64.4)$ & & $86(61.9)$ & $93(66.9)$ & \\
\hline$>0.5$ & $99(35.6)$ & & $53(38.1)$ & $46(33.1)$ & \\
\hline $\mathrm{HT}^{\mathrm{b}}$ & $56(20.1)$ & & $28(20.1)$ & $28(20.1)$ & 1.000 \\
\hline Operative method & & & & & 0.798 \\
\hline $\mathrm{LT}^{\mathrm{c}}$ & $188(67.6)$ & & $93(66.9)$ & $95(68.3)$ & \\
\hline $\mathrm{TT}^{\mathrm{d}}$ & $90(32.4)$ & & $46(33.1)$ & \multirow[t]{2}{*}{$44(31.7)$} & \\
\hline Cervical lymph node & & & & & \\
\hline dissection & $238(85.6)$ & & $119(85.6)$ & $119(85.6)$ & 1.000 \\
\hline
\end{tabular}

a. UGFNA= Ultrasound-guided fine needle aspiration biopsy; b. HT= Hashimoto's thyroiditis; c. LT=Lobectomy; d.TT=Total thyroidectomy.

Table 3 Risk factors of cervical lymph node metastasis in patients who underwent cervical lymph node dissection 


\begin{tabular}{cllll}
\hline Variables & \multicolumn{3}{c}{ Unadjusted $^{c}$ Adjusted } \\
\cline { 2 - 5 } & OR $^{\mathrm{a}}\left(95 \% \mathrm{CI}{ }^{\mathrm{b}}\right)$ & $P$ value & OR $(95 \% \mathrm{CI})$ & $P$ value \\
\hline Age<55, y & $1.749(0.943 \sim 3.243)$ & 0.074 & $1.809(0.937 \sim 3.492)$ & 0.077 \\
Male sex & $3.504(1.984 \sim 6.188)$ & $0.000^{* *}$ & $3.997(2.148 \sim 7.439)$ & $0.000^{* *}$ \\
\hline Multifocality & $1.662(0.829 \sim 3.329)$ & 0.149 & $1.949(0.905 \sim 4.196)$ & 0.088 \\
\hline Capsular invasion & $1.929(0.732 \sim 5.082)$ & 0.178 & $1.218(0.415 \sim 3.572)$ & 0.720 \\
\hline Tumor size>0.5cm & $2.105(1.229 \sim 3.605)$ & $0.006^{* *}$ & $2.319(1.285 \sim 4.186)$ & $0.005^{* *}$ \\
\hline UGFNA ${ }^{c}$ & $1.232(0.729 \sim 2.084)$ & 0.436 & $1.199(0.681 \sim 2.109)$ & 0.530 \\
\hline HT d & $0.692(0.360 \sim 1.328)$ & 0.266 & $0.824(0.401 \sim 1.696)$ & 0.600 \\
\hline
\end{tabular}

a. $\mathrm{OR}=$ odds ratio; $\mathrm{b} . \mathrm{CI}=$ confidence interval; c. UGFNA= Ultrasound-guided fine needle aspiration biopsy; $d$. HT $=$ Hashimoto's thyroiditis. ${ }^{* *}: P<0.01$

\section{Figures}
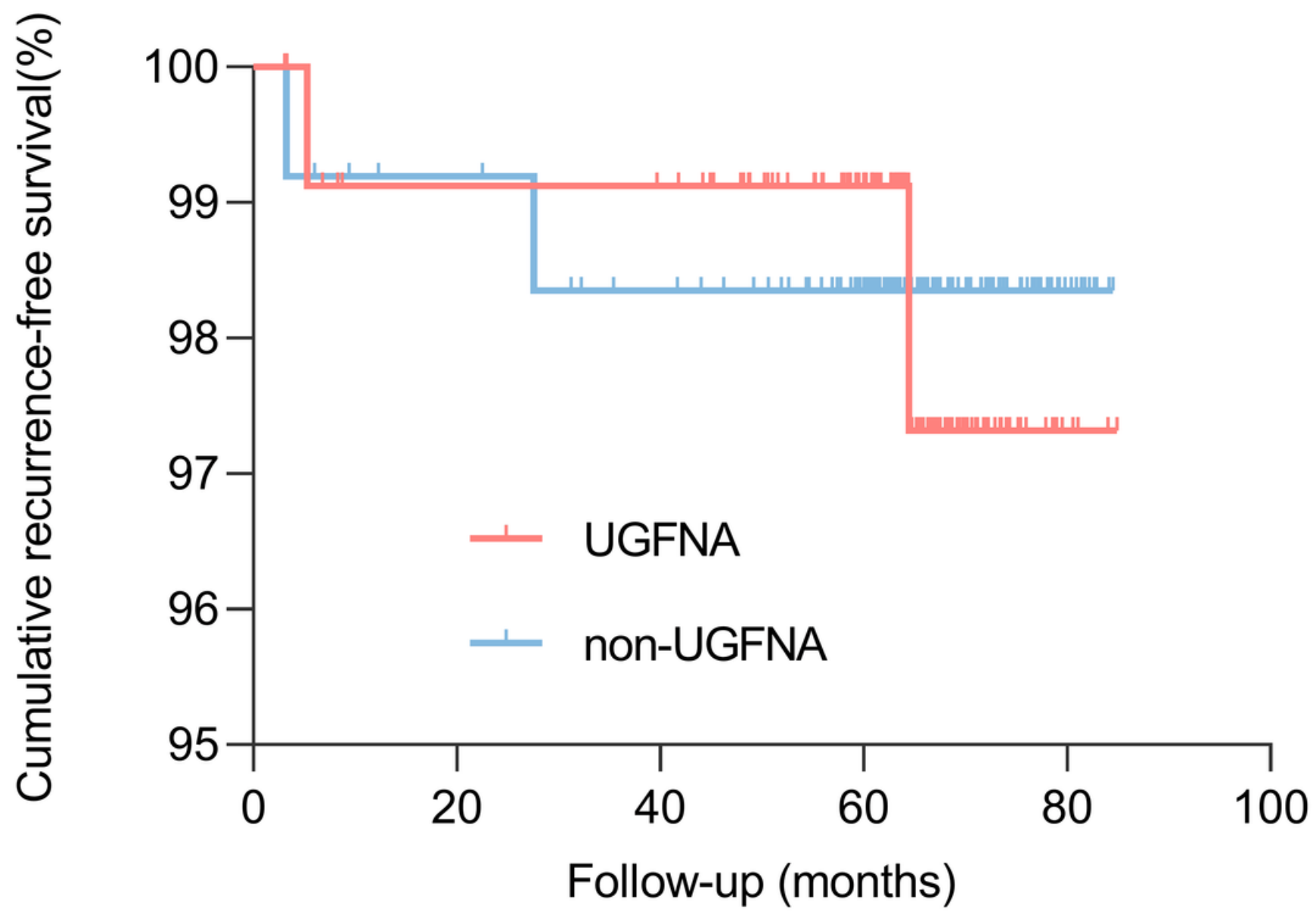

Figure 1 
Kaplan-Meier curves of recurrence-free survival stratified by UGFNA. Cumulative recurrence-free survival rate was calculated by Kaplan-Meier method. Kaplan-Meier curves showed no significant difference between the UGFNA group and non-UGFNA group (Log rank test, $\mathrm{P}=0.927$ ). UGFNA=Ultrasound-guided fine needle aspiration biopsy. 